EPJ Web of Conferences 72, 00012 (2014)

DOI: $10.1051 /$ epjconf / 20147200012

(C) Owned by the authors, published by EDP Sciences, 2014

\title{
Baryon Spectroscopy at ELSA and at MAMI - selected results
}

\author{
B. Krusche $e^{1, a}$ \\ for the CBELSA/TAPS- and A2-collaborations \\ ${ }^{1}$ Department of Physics, University of Basel, CH4056 Basel, Switzerland
}

\begin{abstract}
Spectroscopy of baryons and their excited states plays a key role for our understanding of the strong interaction in the non-perturbative regime. Both, in theory and in experiment, large progress has been made during the last few years. The rapid developments in lattice gauge calculations and the application of the Dyson-Schwinger equation to QCD have opened new perspectives for the interpretation of the excitation spectrum of the nucleon. In parallel, large efforts have been undertaken world-wide, and are still running, to investigate excited nucleon states experimentally, in particular with photoninduced production of mesons. In the present contribution we discuss such experimental programs conducted at the tagged photon beams of the electron accelerators ELSA in Bonn and MAMI in Mainz. These programs are diverse. They include the measurement of cross sections, single- and double polarization observables for single meson production and production of meson pairs off free protons as well as of quasi-free nucleons bound in the deuteron (and sometimes other light nuclei).
\end{abstract}

\section{Introduction}

The excitation spectrum of any bound system of particles is expected to reflect the properties of the underlying interaction. Well-known examples are atomic spectra and nuclear excitation schemes which have been used to study the properties of the Coulomb interaction and of the residual strong interaction between nucleons in much detail. In the latter case, such studies have revealed very successfully the relevant effective degrees of freedom that allow us to characterize the different types of excited states like single-particle excitations or collective vibrations and rotations.

A similar approach for the relation between the excited states of nucleons and the fundamental properties of Quantum Chromodynamics (QCD) had so far much more limited success. Simplifying models use constituent quarks as effective degrees of freedom. However, clustering of the quarks into di-quark structures, additional degrees of freedom due to excited flux tubes, and molecule-like states arising from coupled channel dynamics have also been suggested [1]. Such different effective degrees of freedom are reflected in the predicted excitation patterns, but so far agreement between all model predictions and the experimental data base was not good enough to draw any final conclusions.

Recent progress, however, is significant. On the theory side, fully relativistic quark model approaches have been developed [2], and also the application of the Dyson-Schwinger approach to QCD has led to promising new results (see e.g. [3-5]). The advances in lattice gauge calculations

\footnotetext{
ae-mail: Bernd.Krusche@unibas.ch
} 
and their combination with the methods of chiral perturbation theory for the extrapolation to physical quark masses allowed first predictions of the excitation spectrum based on unquenched lattice results [6]. Interestingly, they basically 're-discovered' the $\mathrm{SU}(6) \otimes \mathrm{O}(3)$ excitation structure of the nucleon with a level counting consistent with the standard non-relativistic quark model. However, one should keep in mind that these calculations are still at a very early stage (they used a pion mass of $400 \mathrm{MeV}$, while groundstate predictions nowadays can be directly made at the physical point).

Efforts on the experimental side were comparably intensive. They aimed in particular at a much less biased data base for the excited nucleon states. A few years ago the listing of $N^{\star}$ (isospin $I=1 / 2$ ) and $\Delta^{\star}(I=3 / 2)$ resonances in the Review of Particle Physics (PDG) included only states that had been observed in inelastic pion scattering reactions, only in the most recent update [7] states 'established' by observations in photon induced reactions were included. Possible bias from elastic pion scattering is obvious. States that do not couple significantly to $N \pi$ are strongly suppressed, in excitation as well as in decay. This problem becomes more severe for higher excitations energies. The higher the excitation energy, the larger grows the phase space for decays involving heavier mesons, and, maybe even more important, the larger becomes the probability for cascade decays involving an intermediate excited state. This is not only a question of phase space, it is also related to the internal structure of resonances. It is for example a plausible conjecture that resonances, which have more than one oscillator excited, will tend to de-excite them step-by-step, so that entire multiplets of states may be difficult to observe in single meson production reactions.

The experimental efforts during the last decade, which are still continuing, tried to remove such bias by a large-scale investigation of meson-photoproduction reactions using many different final states, among them also meson pairs (mainly $\pi \pi, \pi \eta$ ) aiming at cascade decays. In comparison to inelastic pion scattering, photoproduction reactions are complicated by the spin degree of freedom of the incident photon and by the isospin dependence and non-conservation of the electromagnetic interaction. It can be shown that the complete determination of the amplitudes of photoproduction of a single pseudo-scalar meson requires the measurement of at least eight carefully chosen observables [8] as function of two independent kinematic variables, while for photoproduction of pseudo-scalar meson pairs [9] the measurement of eight observables as function of five kinematic parameters allows only the extraction of the magnitude of the amplitudes and in total 15 measurements would be required to fix in addition the phases. Investigation of the isospin structure of the electromagnetic excitations requires furthermore the measurement of two (for isoscalar mesons like $\eta, \eta^{\prime}$ ) or three (for isovectors like $\pi$ ) independent isospin amplitudes. The experimental study of such reactions therefore requires not only the measurement of angular distributions and (for the three-body final states) of invariant mass distributions but also the exploration of polarization observables and this not only for measurements off free protons but also for quasi-free neutrons.

The Bonn ELSA and Mainz MAMI experiments can deliver linearly and circularly polarized photon beams and are equipped with solid-state cryo-targets that provide longitudinally or transversely polarized protons or deuterons. Both experimental programs cover all aspects discussed above: the measurement of many different final states including meson pairs like $\pi \pi$ and $\pi \eta$, the measurement of all single and double polarization observables accessible with polarized beams or/and polarized targets and (in Mainz) also the measurement of some observables involving recoil polarization, and the use of protons and neutrons as initial state nucleons. Large part of the experiments is complementary to other tagged photon facilities like for example CLAS at Jlab, which is optimized for the detection of charged particles and can measure only final states with not more than one neutral particle (neutral meson decaying into photons, neutron) using missing mass techniques. The experiments discussed here are also sensitive to final states with two or more neutral mesons even when they are produced off the neutron. 


\section{Experiments}

The two experiments are similar in many aspects. One of the main differences lies in the accelerator specifications. The stretcher synchrotron ELSA can deliver electron beams with energies up to $3.5 \mathrm{GeV}$ of relatively low intensities (order of $\approx 1 \mathrm{nA}$ ), while the cascaded race-track microtron [11] in Mainz reaches energies up to $1.5 \mathrm{GeV}$ with intensities up to $\approx 100 \mu \mathrm{A}$, far beyond what is needed $(\approx$ few tens of $\mathrm{nA}$ ) for tagged-photon beams (the accelerator serves also for electron scattering which can use much higher intensities).

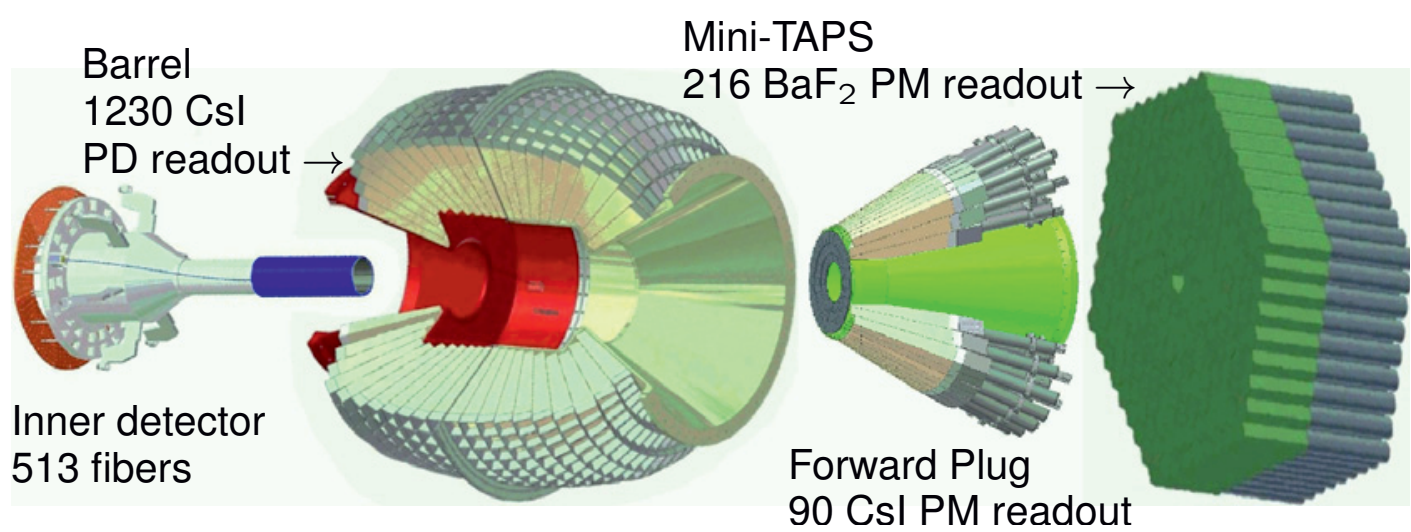

Figure 1. Setup of the Crystal Barrel/TAPS experiment at ELSA. Different components are indicated, the beam enters from the left hand side.

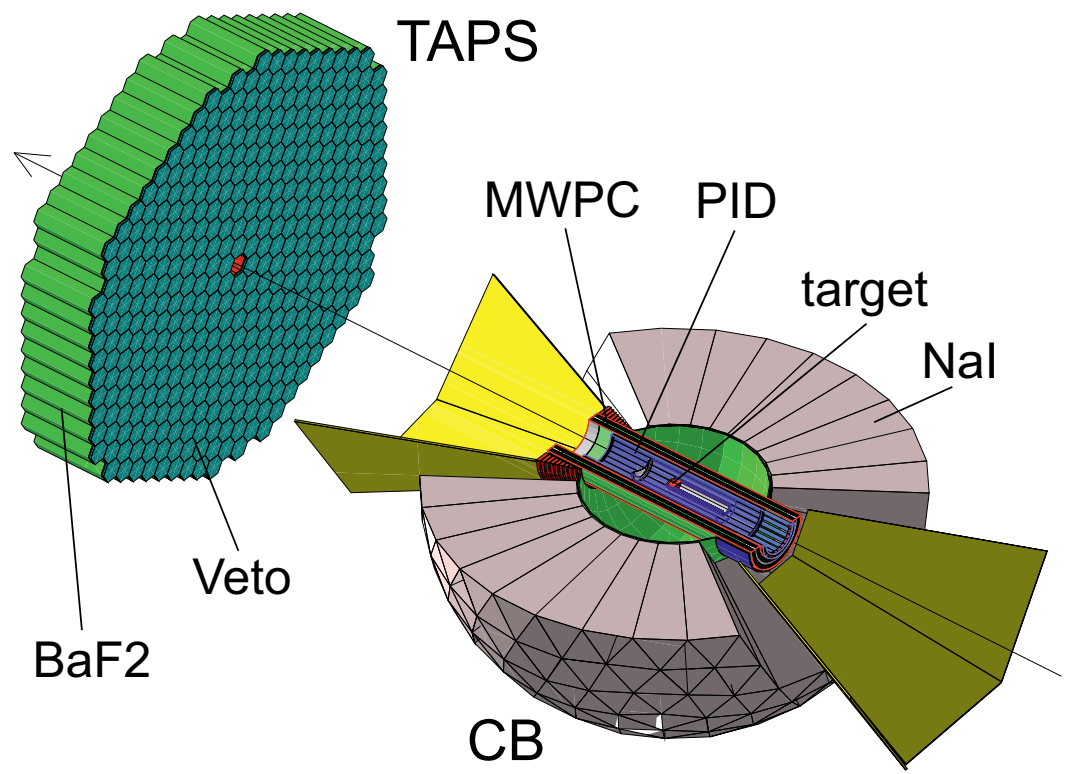

Figure 2. Setup of the Crystal Ball/TAPS experiment at MAMI. Only the lower hemisphere of the Crystal Ball is shown. Beam enters from right hand side. PID: (charged) particle identification detector made of scintillator strips, MWPC: multi-wire chambers. 
Tagged photon beams are produced by scattering the electrons in a radiator to produce bremsstrahlung, which is energy tagged by a momentum analysis of the scattered electrons in magnetic spectrometers. The tagging device used in Mainz is described in detail in [12] (and Refs. therein). Both accelerators can deliver longitudinally polarized electron beams, which are used to produce circularly polarized photon beams. Linearly polarized photon beams are produced with coherent bremsstrahlung from a diamond lattice. This method is discussed for the ELSA setup in [13]. Polarized target nucleons are available from buthanol targets in frozen-spin mode, the device used at MAMI is described in [14]. The definition of the beam/target related polarization observables is summarized in Fig. 3.
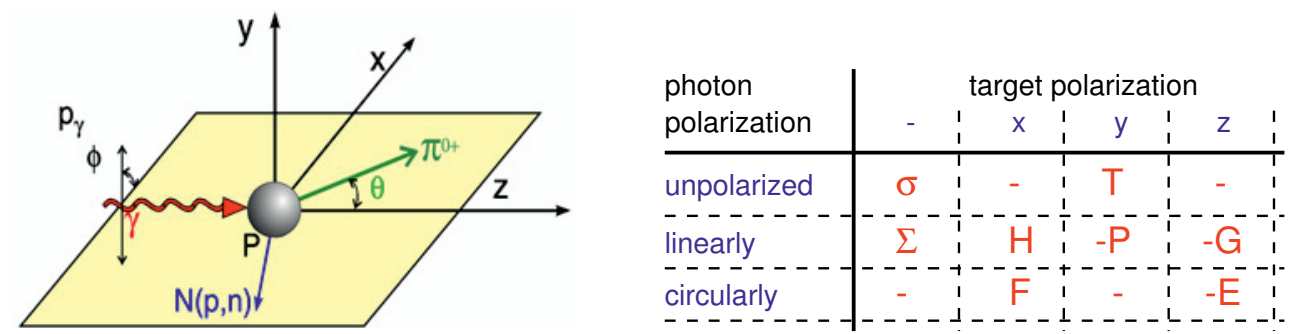

$$
\begin{aligned}
\frac{d \sigma}{d \Omega}=\frac{d \sigma_{0}}{d \Omega}\left\{1-P_{l} \Sigma \cos (2 \phi)\right. & +P_{x}\left[-P_{l} H \sin (2 \phi)+P_{c} F\right] \\
& -P_{y}\left[-T+P_{l} P \cos (2 \phi)\right] \\
& \left.-P_{z}\left[-P_{l} G \sin (2 \phi)+P_{c} E\right]\right\}
\end{aligned}
$$

Figure 3. Definition of the beam/target polarization related observables for photoproduction of single, pseudoscalar mesons.

The reaction products are detected with almost $4 \pi$ covering electromagnetic calorimeters, which are schematically shown in Figs. 1,2. The large angle coverage of these devices and their good detection properties for photons and neutrons (but also for charged particles) is the basis of the versatile experimental programs. In both cases the largest part of the solid angle is covered by more or less spherical calorimeter parts - the Crystal Barrel [16] composed of CsI scintillators at ELSA and the Crystal Ball [15] made of NaI modules at MAMI - and a forward scintillator wall of $\mathrm{BaF}_{2}$ crystals from the TAPS detector [17]. The calorimeters are supplemented by detectors for charged particle identification and tracking mounted around the targets (which are placed in the center of the Barrel/Ball calorimeters) and in front of the crystals of the TAPS components. More details about the setups and data analysis procedures are given in the references cited below for the examples of experimental results.

\section{Experimental results}

In this section we will summarize some final and preliminary results from the experimental programs at ELSA and MAMI. We start with the most intensively studied reaction channel - photoproduction of $\pi^{0}$-mesons - for which the measurement of polarization observables has recently contributed even at low excitation energies significantly to the understanding of resonance contributions. For this reaction we will also discuss first, preliminary results for cross sections and polarization observables for reactions off quasi-free neutrons. Photoproduction of isoscalar mesons like $\eta, \eta^{\prime}$ is important for the 
investigation of $N^{\star}$ resonances and the isospin decomposition of their electromagnetic excitations. In particular the reaction $\gamma n \rightarrow n \eta$ has attracted much interest due to the observation of a narrow structure, that was not observed for the proton target. Finally, we will discuss some results for the photoproduction of pseudoscalar mesons pairs. In case of pion pairs polarization observables, which exist only for three-body final states, have been recently studied for different isospin channels. Finally, also the study of the production of $\eta \pi$ pairs has been extended to different isospin channels.

\subsection{Photoproduction of $\pi^{o}$-mesons}

Historically, reactions involving pion scattering or single pion production reactions where the backbone for the study of nucleon resonances. In photoproduction the $N \pi^{0}$ channel is very interesting because it has much less important contributions from non-resonant backgrounds (pion-pole, KrollRuderman terms etc.) than reactions involving charged pions to which the incident photon can couple directly. The previously available data base had been analyzed with different reaction models and partial wave analyses like e.g. MAID, SAID, and the Bonn-Gatchina model (BnGn) [19-21]. However, already the results for the first double polarization observable $(G)$ measured at ELSA [23] made a readjustment of the model fits necessary, surprisingly already for very low incident photon energies. The largest effects were found for the $E_{0+}$ and $E_{2-}$ partial waves involving excitation e.g. of the $S_{11}(1535)$ and $D_{13}(1520)$ nucleon resonances.
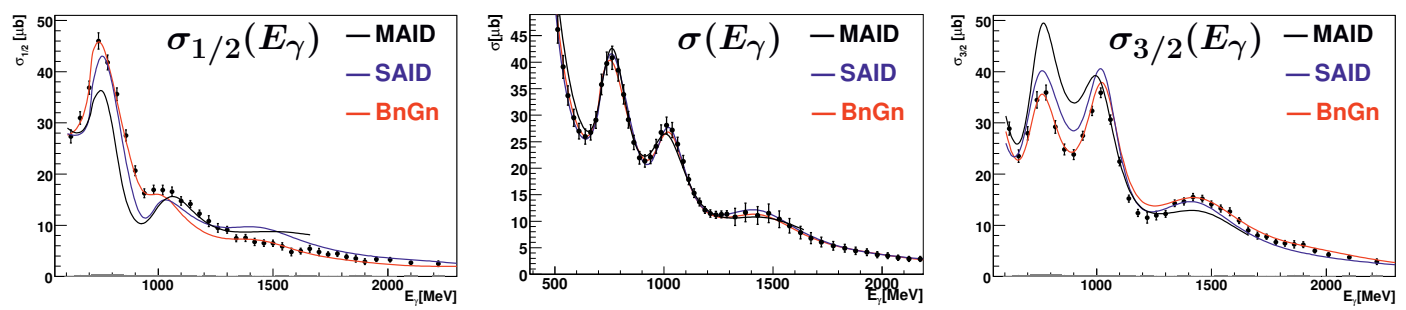

Figure 4. Split of the total cross section (center) of $\gamma p \rightarrow p \pi^{0}$ into the helicity-1/2 (left hand side) and helicity-3/2 (right hand side) components [18] (ELSA). Theory curves from MAID [20], SAID [19], and BnGn [21].

Here, we discuss another polarization degree of freedom, namely the helicity decomposition of the total cross section (related to the double polarization observable $E$ ). This is measured with a circularly polarized photon beam on a longitudinally polarized target. Parallel alignment of photon and nucleon spin corresponds to a spin projection on the beam axis of $3 / 2$ (only possible for nucleon resonances with $J \geq 3 / 2$ ), while with antiparallel alignment also $J=1 / 2$ states may be excited. The unpolarized total cross section and the $\sigma_{1 / 2}$ and $\sigma_{3 / 2}$ components measured at ELSA [18] are compared in Fig. 4 to model predictions. All models agree with the unpolarized cross section (they have been fitted to it), but the spilt into helicity components is quite different in the models. Altogether, it is obvious, that further measurements of polarization observables are necessary for a convergence of the different analyses approaches.

As an example for the already achieved quality of such data we show in Fig. 5 some preliminary data measured at ELSA for the observables $T$ (target asymmetry), $P$ (recoil polarization), and the double polarization observable $H$, which has been measured for the first time for this reaction.

A complete determination of the amplitudes involved in photoproduction of a pseudo-scalar meson requires also the measurement of observables involving the polarization state of the recoil nucleon. Measurement of the recoil nucleon polarization is difficult, it requires scattering of the final state nucleons off nuclei which reduces largely the statistical quality of data. For the single polarization 


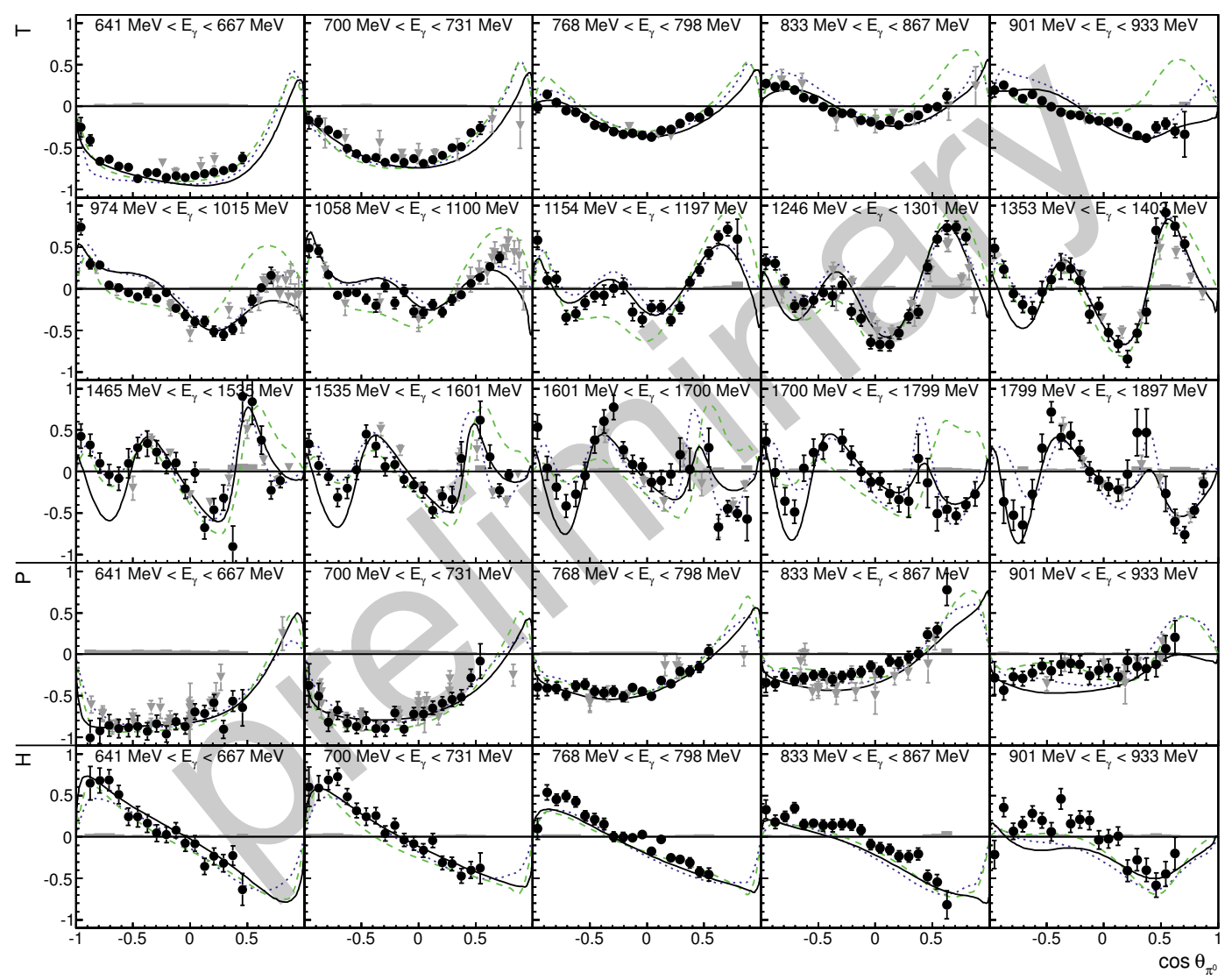

Figure 5. Examples for the polarization observables $T$ (target asymmetry, transversely polarized target), $P$ (recoil polarization) and $H$ both measured as double polarization observables with transversely polarized target and linearly polarized beam) (ELSA). Black dots: present results, grey triangles: previous results for $T$ and $P$ [24], curves: MAID (dashed) [20], SAID (dotted) [19], and BnGn (solid) [21]

observable $P$ this can be avoided, because as indicated in Fig. 3, it is also accessible from a (more simple) double polarization measurement with linearly polarized beam and transversely polarized target. However, double polarization observables involving recoil polarization are also needed. Here, recently progress has been made at the MAMI facility using a novel large-acceptance recoil nucleon polarimeter based on the scattering process off Carbon nuclei. As a first test the observable $C_{x}^{\star}$ involving circularly polarized photons and the measurement of the recoil proton polarization has been measured for the $\gamma p \rightarrow p \pi^{0}$ reaction [25].

Much less data is so far available for the photoproduction of pions off neutrons. The isospin decomposition of the cross section is so far entirely based on the measurement of the $\gamma n \rightarrow p \pi^{-}$ reaction off quasi-free neutrons [26, 27]. However, in this reaction channel contributions from nonresonant background terms are much stronger than for $\gamma n \rightarrow n \pi^{0}$. Preliminary results for a first measurement of the latter reaction in quasi-free kinematics using a deuteron target are available from MAMI. The final state invariant mass $W$ has been deduced from the completely determined reaction kinematics as discussed in [28], so that effects from nuclear Fermi motion were eliminated. 

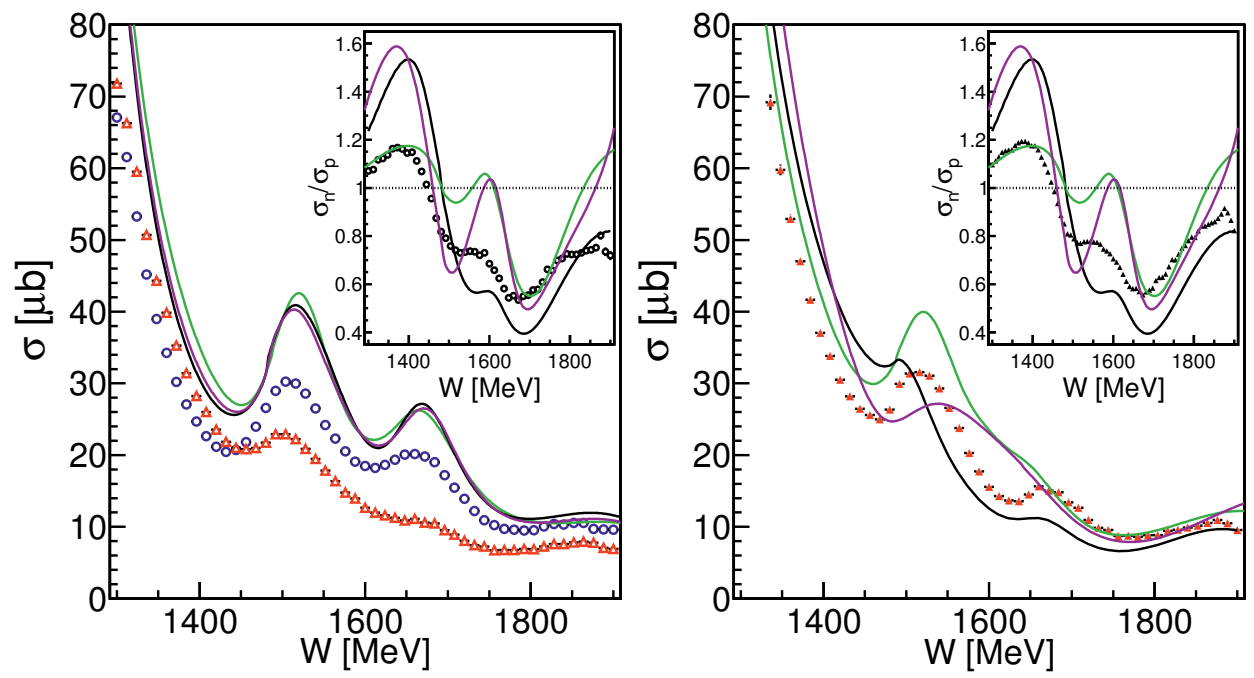

Figure 6. Total cross sections of $\gamma N \rightarrow N \pi^{o}$ for photoproduction of quasi-free nucleons bound in the deuteron (MAMI). Right hand side: quasi-free proton (blue circles) and quasi-free neutron (red triangles) compared to model predictions for free proton target (black: SAID [19], green MAID [20], magenta BnGn [21, 22]). Right hand side: FSI corrected (see text) results for neutron target compared to model predictions for free neutron. Inserts: neutron/proton ratios. All results preliminary.
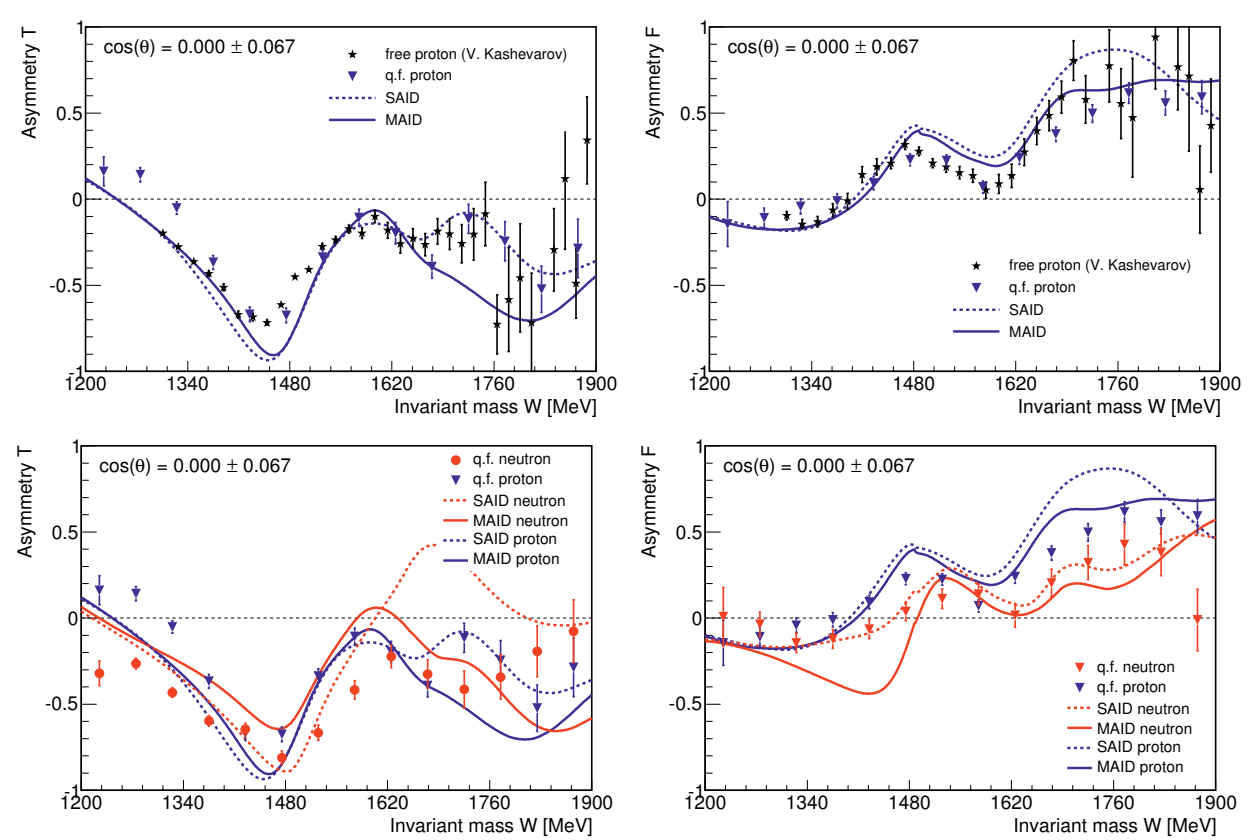

Figure 7. Polarization observables for quasi-free protons and neutrons for the $\gamma N \rightarrow N \pi^{o}$ reaction with transversely polarized target and circularly polarized beam (MAMI). Left hand side: target asymmetry $T$, right hand side: observable $F$, upper part: free (black stars) and quasifree (blue triangles) protons, bottom: quasi-free protons (blue triangles) and quasi-free neutrons (red spheres). All data very preliminary. 
Total cross sections are compared in Fig. 6 to model predictions. The left hand side of the figure shows a comparison of the quasi-free proton and neutron cross sections, which are quite different, indicating different nucleon resonance contributions. Also shown at the left hand side are the results for the MAID [20], SAID [19], and BnGn [21] analyses for the free proton. The three theory curves agree among each other (because they have all been fitted to the same data base) but not with the quasifree proton data. This is evidence for final state interaction (FSI) effects, which is not surprising, FSI corrections were also necessary for the $\gamma n \rightarrow p \pi^{-}$channel and at lower incident photon energies, in the excitation range of the $\Delta$ resonance, substantial effects had also already been reported for the $N \pi^{0}$ final state [29]. Model estimates for FSI at higher incident photon energies are not yet available for this channel, however, as a first approximation one can assume, that the effects are similar for bound protons and bound neutrons. With this assumptions one can derive $W$ and angle dependent correction factors from the comparison of free and quasi-free proton data and apply them to the neutron data. The result is shown at the right hand side of Fig. 6 and compared to the MAID [20], SAID [19], and BnGn [22] model predictions. The results from the three models do not at all agree among each other, indicating that the analysis of the $p \pi^{-}$final state is not sufficient to fix the isospin decomposition of the reaction. None of the predictions is in close agreement with the data.

The measurement of single and double polarization observables for $\pi^{0}$ production off quasi-free nucleons has also been initiated. Very preliminary results for the target asymmetry $T$ and the double polarization observable $F$ measured at MAMI are shown in Fig. 7. In this case, agreement between free and quasi-free data is better, FSI effects on this polarization observables seem to be smaller than for absolute cross sections.

\subsection{Photoproduction of isoscalar mesons - $\eta$ and $\eta^{\prime}$}

Photoproduction of the 'heavy', isoscalar mesons $\eta$ and $\eta^{\prime}$ is interesting for two aspects. Only $N^{\star}$ resonances couple to this decay channel and due to the large mass of this mesons only a few partial waves contribute at medium incident photon energies. Experimental progress has been substantial over the last few years. Total cross sections and angular distributions for $\gamma p \rightarrow p \eta$ and $\gamma p \rightarrow p \eta^{\prime}$ have been studied or are under analysis at ELSA [30-32] and MAMI [33]. First results for polarization observables for this reactions will follow soon (data under analysis).

Since these mesons couple only to $N^{\star}$ resonances, the isospin composition of the electromagnetic excitations is in the focus. For both mesons it has been found [34-36] that for quasi-free production of nucleons bound in the deuteron FSI effects are negligible.

The $\gamma n \rightarrow n \eta$ reaction has attracted a lot of interest because an unexpected narrow structure has been observed in the excitation function [34, 38, 39]. More recent measurements at MAMI [36, 37] have confirmed this structure with much improved statistical significance not only for neutrons bound in the deuteron but also for the ${ }^{3} \mathrm{He}$ target (see Fig 8). In spite of the much larger effects from Fermi motion, the $W$ reconstructed data for both targets agree on the main parameters of this structure, only the absolute scale of the data differ. The quasi-free cross sections for neutrons as well as for protons measured from the ${ }^{3} \mathrm{He}$ target are suppressed by $\approx 25 \%$ with respect to the results from the deuteron target. This indicates substantial nuclear effects (FSI) for the helium nuclei, which seem to be more or less identical for $\gamma p \rightarrow p \eta$ and $\gamma n \rightarrow n \eta$. The structure can be phenomenologically fitted with a BreitWigner curve with position $W_{R}=(1670 \pm 5) \mathrm{MeV}$, a width of $\Gamma_{R}=(30 \pm 15) \mathrm{MeV}$ and a strength of $\sqrt{b_{\eta}} A_{1 / 2}^{n}=(12.3 \pm 0.8) 10^{-3} \mathrm{GeV}^{-1 / 2}$ [36]. It's nature is not yet understood. The present measurements have produced precise differential cross sections, which show a non-trivial angular dependence of the structure [36, 37]; it almost disappears for extreme forward and backward angles. Currently, first measurements of the helicity dependence of the cross section and the polarization observables $T$ and $F$ are under analysis (MAMI). 

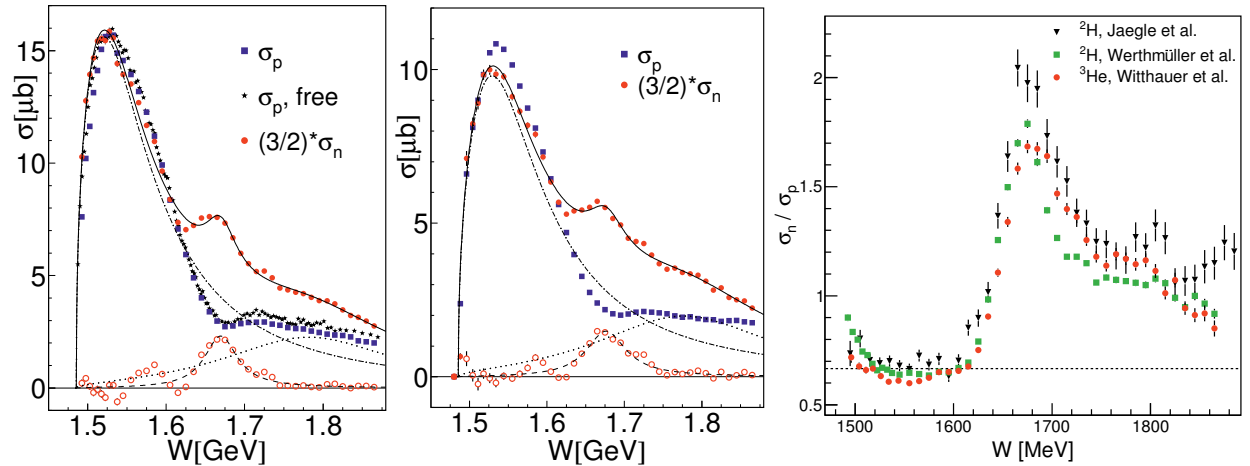

Figure 8. Quasi-free excitation functions for $\gamma N \rightarrow N \eta$. Left hand side: deuteron [36] and free proton [33] targets; quasi-free proton (blue squares), free proton (black stars), and quasi-free neutron (red circles). Curves: fits (see text): $S_{11}$ contribution (dash-dotted), phenomenological background (dotted), narrow structure (dashed), sum of all (solid). Open red circles: data after subtraction of $S_{11}$ and background fit. Center: same for ${ }^{3} \mathrm{He}$ target [36, 37]. Right hand side: ratio of neutron/proton excitation functions [34] (ELSA), [36, 37] (MAMI).

\subsection{Photoproduction of $\eta \pi$ pairs}

Photoproduction of meson pairs should give access to excited nucleon states which have no significant decay branching ratios directly to the nucleon ground state but decay preferentially via cascades involving intermediate excited states. The best studied double-meson final state are pion pairs (see 3.4). More recently also the $\eta \pi$ final state attracted much interest. Total cross sections, invariant mass
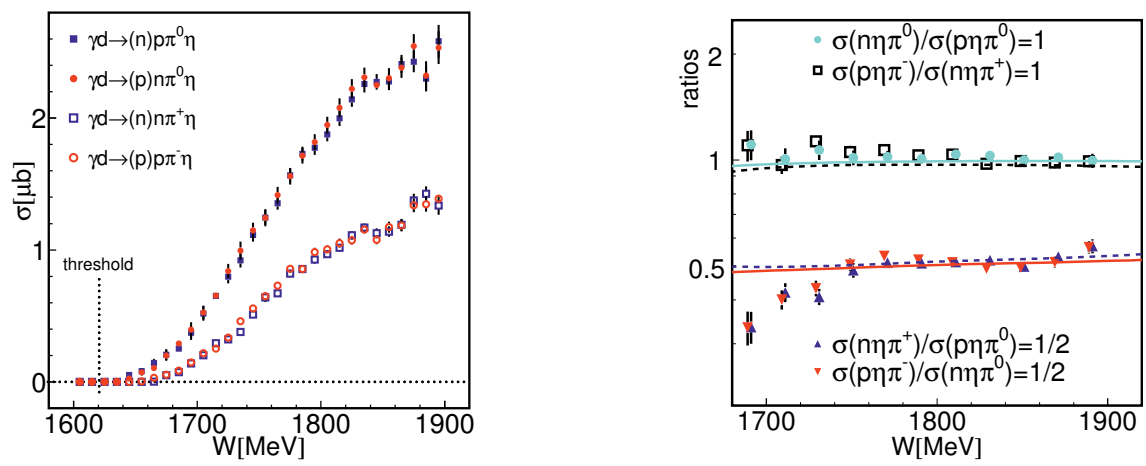

Figure 9. Quasi-free excitation functions for $\gamma N \rightarrow N \eta \pi$ measured with a deuteron target. Left hand side: total cross sections, right hand side: cross section ratios. All data preliminary. Model curves from [46].

distributions, and also some polarization observables have been measured for $\gamma p \rightarrow p \pi^{0} \eta$ [40-47]. This decay channel is very selective. The $\eta$-meson is isoscalar, so that nucleon resonances can only emit it in $N^{\star} \rightarrow N^{(\star)}$ or $\Delta^{\star} \rightarrow \Delta^{(\star)}$ transitions. The results [43, 46] suggested a dominant contribution from the $D_{33}(1700) \rightarrow \eta P_{33}(1232) \rightarrow \eta \pi N$ cascade in the threshold region. When the reaction is indeed dominated by this cascade simple predictions can be made for the cross section ratios of the different charge states. The electromagnetic helicity couplings for the excitation of $\Delta$ resonances are identical for protons and neutrons and from the Clebsch-Gordon coefficients of the different hadronic decays one arrives at: $\sigma\left(\gamma p \rightarrow \eta \pi^{0} p\right)=\sigma\left(\gamma n \rightarrow \eta \pi^{0} n\right)=2 \sigma\left(\gamma p \rightarrow \eta \pi^{+} n\right)=2 \sigma\left(\gamma n \rightarrow \eta \pi^{-} p\right)$, while 
for the photoexcitation of an $N^{\star}$ resonance the factors 2 would be $1 / 2$ and the cross section ratios for neutron and proton targets could be anything. Data for all four isospin channels has been measured at MAMI. Preliminary results for total cross sections and their ratios are summarized in Fig. 3.3. They are in excellent agreement with the above expectations. The absolute scale of the cross sections for the quasi-free proton bound in the deuteron are suppressed with respect to the free proton by roughly $25 \%-30 \%$, so that significant FSI effects are observed.

\subsection{Photoproduction of pion pairs}

Photoproduction of pion pairs has been previously studied at ELSA and MAMI for different final states, investigating total cross sections, invariant mass distributions, and partly also the helicity dependence of the cross section [50-63]. However, the complicated amplitude structure of this reaction requires the investigation of further polarization observables. Such a program is currently active at ELSA and MAMI. First results are available for the beam-helicity asymmetry $I^{\odot}(\Phi)=\left(d \sigma^{+}-d \sigma^{-}\right) /\left(d \sigma^{+}+\sigma^{-}\right)$, where $d \sigma^{ \pm}$are the cross sections for the two polarization states of a circularly polarized beam on an unpolarized target. The angle $\Phi$ is defined as shown in Fig 9 between
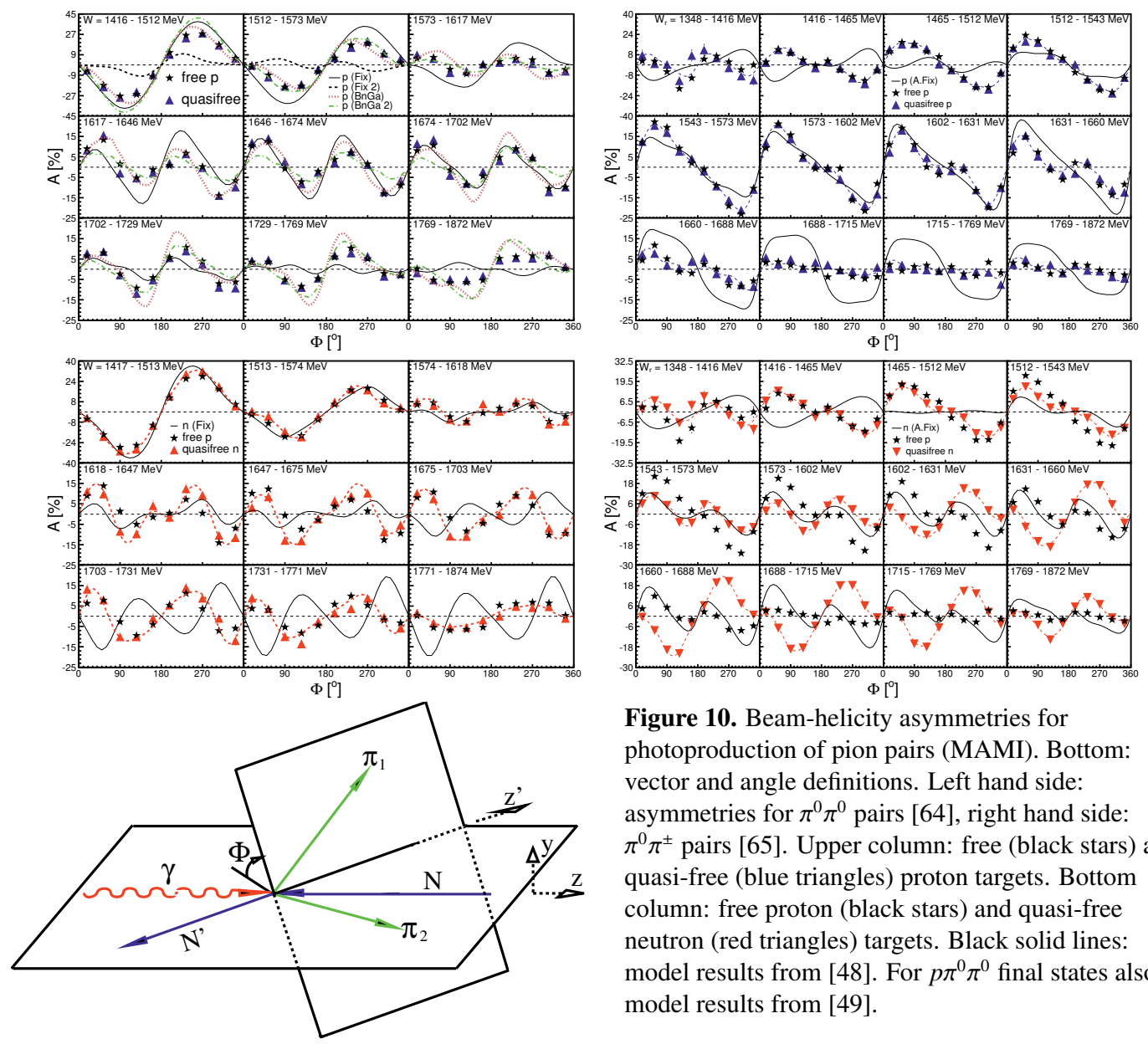

Figure 10. Beam-helicity asymmetries for photoproduction of pion pairs (MAMI). Bottom: vector and angle definitions. Left hand side: asymmetries for $\pi^{0} \pi^{0}$ pairs [64], right hand side: $\pi^{0} \pi^{ \pm}$pairs [65]. Upper column: free (black stars) and quasi-free (blue triangles) proton targets. Bottom column: free proton (black stars) and quasi-free neutron (red triangles) targets. Black solid lines: model results from [48]. For $p \pi^{0} \pi^{0}$ final states also model results from [49]. 
two planes defined by the incident photon and the three final state hadrons. For the examples shown in Fig. 9 the reaction plane is defined by the photon and the recoil nucleon, while the production plane is spanned by the two pions, ordered such that $m\left(\pi_{1}, N^{\prime}\right) \geq m\left(\pi_{2}, N^{\prime}\right)$ where $m\left(\pi_{i}, N^{\prime}\right)$ are the invariant masses of the nucleon-pion pairs. This observable has been measured at MAMI for the final states $p \pi^{0} \pi^{0}, n \pi^{0} \pi^{0}, n \pi^{0} \pi^{+}$, and $p \pi^{0} \pi^{-}$. For the neutral as well as for the mixed-charged pion pairs results for the free and quasi-free proton are in excellent agreement, demonstrating that the quasi-free neutron data can be interpreted as good approximation of free neutron data. Agreement with model predictions [48] is not yet good; such data will certainly better constrain future analyses. Data for other polarization observables (e.g. target asymmetry $T$, and $F$ asymmetry (MAMI) and $G$ (ELSA)) are also under analysis.

\section{Acknowledgements}

The results summarized in this work are part of the experimental programs of the CBELSA/TAPS (ELSA) and A2 (MAMI) collaborations. The unpublished, preliminary results are parts of the PhD theses of J. Hartmann (Bonn) and M. Dieterle, A. Käser, and Th. Strub (Basel). This work was supported by Schweizerischer Nationalfonds (200020-132799,121781,117601,113511), Deutsche Forschungsgemeinschaft (SFB 443, SFB/TR 16), DFG-RFBR (Grant No. 05-02-04014), UK Science and Technology Facilities Council, (STFC 57071/1, 50727/1), European Community-Research Infrastructure Activity (FP6), the US DOE, US NSF and NSERC (Canada).

\section{References}

[1] C. Amsler, B. Krusche, T. DeGrand (Quark Model review), in J. Beringer et al., Phys. Rev. D 86, 010001 (2012).

[2] W. Plessas, Mod. Phys. Lett. A 28, 1360022 (2013).

[3] Ch. Chen et al., Few Body Syst. 53, 293 (2012).

[4] G. Eichmann Prog. Part. Nucl. Phys. 67, 234 (2012).

[5] I.G. Aznauryan et al., Int. J. Mod. Phys. E 22, 1330 (2013).

[6] R.G. Edwards et al., Phys. Rev. D 84, 074508 (2011).

[7] J. Beringer et al., Phys. Rev. D 86, 010001 (2012).

[8] W.T. Chiang and F. Tabakin, Phys. Rev. C 55, 2054 (1997).

[9] W. Roberts and T. Oed, Phys. Rev. C 71, 055201 (2005).

[10] W. Hillert, Eur. Phys. J. A 28, 139 (2006).

[11] K.-H. Kaiser et al., Nucl. Instr. Meth. A 593, 159 (2008).

[12] J.C. McGeorge et al., Eur. Phys. J. A 37, 129 (2008).

[13] D. Elsner et al., Eur. Phys. J. A 39, 373 (2009).

[14] A. Thomas et al., Phys. of Particles and Nuclei 44, 964 (2013).

[15] A. Starostin et al., Phys. Rev. C 64, 055205 (2001).

[16] E. Aker et al., Nucl. Instr. and Meth. A 321, 69 (1992).

[17] A.R. Gabler et al., Nucl. Instr. and Meth. A 346, 168 (1994).

[18] M. Gottschall et al., Phys. Rev. Lett. 112, 012003 (2014).

[19] R.L. Workman et al., Phys. Rev. C 86, 015202 (2012).

[20] D. Drechsel, S.S. Kamalov, L.Tiator, Eur. Phys. J. A 34, 69 (2007).

[21] A.V. Anisovich et al., Eur. Phys. J. A 44, 203 (2010).

[22] A.V. Anisovich et al., Eur. Phys. J. A 49, 67 (2013). 
[23] A. Thiel et al., Phys. Rev. Lett. 109, 102001 (2012).

[24] W. Briscoe et al., GWU analysis center, (2013).

[25] M.H. Sikora et al., Phys. Rev. Lett. 112, 022501 (2014).

[26] W. Chen et al., Phys. Rev. Lett. 103, 012301 (2009).

[27] W. Chen et al., Phys. Rev. C 86, 015206 (2012).

[28] B. Krusche, Eur. Phys. J. Special Topics 198, 199 (2011).

[29] B. Krusche and S. Schadmand, Prog. Part. Nucl. Phys. 51, 399 (2003).

[30] V. Crede et al., Phys. Rev. Lett. 94, 012004 (2005).

[31] O. Bartholomy et al., Eur. Phys. J. A 33, 133 (2007).

[32] V. Crede et al., Phys. Rev. C 80, 055202 (2009).

[33] E.F. McNicoll et al., Phys. Rev. C 82, 035208 (2010).

[34] I. Jaegle et al., Eur. Phys. J. A 47, 89 (2011).

[35] I. Jaegle et al., Eur. Phys. J. A 47, 11 (2011).

[36] D. Werthmüller et al., Phys. Rev. Lett. A 49, 154 (2013).

[37] L. Witthauer et al., Eur. Phys. J. 111, 232001 (2013).

[38] V. Kuznetsov et al., Phys. Lett. B 647, 23 (2007).

[39] I. Jaegle et al., Phys. Rev. Lett. 100, 252002 (2008).

[40] T. Nakabayashi et al., Phys. Rev. C 74035202 (2006).

[41] J. Ajaka et al., Phys. Rev. Lett. 100, 052003 (2008).

[42] I. Horn et al., Phys. Rev. Lett. 101, 202002 (2008).

[43] I. Horn et al., Eur. Phys. J. A 38, 173 (2008).

[44] E. Gutz et al., Eur. Phys. J. A 35, 291 (2008).

[45] E. Gutz et al., Phys. Lett. B 687, 11 (2010).

[46] V. Kashevarov et al., Eur. Phys. J. A 42, 141 (2009).

[47] V. Kashevarov et al., Phys. Lett. B 693, 551 (2010).

[48] A. Fix and H. Ahrenhövel, Eur. Phys. J. A 25, 115 (2005).

[49] A.V. Anisovich et al., Eur. Phys. J. A 48, 15 (2012).

[50] A. Braghieri et al., Phys. Lett. B 363, 46 (1995).

[51] F. Härter et al., Phys. Lett. B 401, 229 (1997).

[52] A. Zabrodin et al., Phys. Rev. C 55, R1617 (1997).

[53] A. Zabrodin et al., Phys. Rev. C 60, 055201 (1999).

[54] B. Krusche et al., Eur. Phys. J. A 6, 309 (1999).

[55] M. Wolf et al., Eur. Phys. J. A 9, 5 (2000).

[56] V. Kleber et al., Eur. Phys. J. A 9, 1 (2000).

[57] W. Langgärtner et al., Phys. Rev. Lett. 87, 052001 (2001).

[58] J. Ahrens et al., Phys. Lett. B 551, 49 (2003).

[59] M. Kotulla et al., Phys. Lett. B 578, 63 (2004).

[60] A.V. Sarantsev et al., Phys. Lett. B 659, 94 (2008).

[61] U. Thoma et al., Phys. Lett. B 659, 87 (2008).

[62] D. Krambrich et al., Phys. Rev. Lett. 103, 052002 (2009).

[63] F. Zehr et al., Eur. Phys. J. A 48, 98 (2012).

[64] M. Oberle et al., Phys. Lett. B 721, 237 (2013).

[65] M. Oberle et al., submitted to Eur. Phys. J. A (2014). 